\title{
Effects of the ghrelin receptor agonist anamorelin on lean body mass in cancer patients with cachexia; results from a Phase II randomized, double blind, multicenter study
}

\author{
Jose M Garcia ${ }^{1,2}$, Ying Yan ${ }^{3}$, Elizabeth Manning-Duus ${ }^{3 *}$, John Friend ${ }^{3}$ \\ From Metabolism, Diet and Disease 2014: Cancer and metabolism \\ Washington DC, USA. 28-30 May 2014
}

\section{Background}

Cancer anorexia-cachexia is a frequent, debilitating and life-threatening condition in which altered metabolism and reduced food intake contribute to weight loss (mainly due to lean body mass [LBM] loss), which cannot be reverted by conventional nutritional support. Safe/effective treatments for cancer cachexia remain an unmet need. The hunger hormone ghrelin has been shown to activate key pathways in the regulation of body composition. Anamorelin (ANAM) is a novel, selective, oral ghrelin receptor agonist with appetiteenhancing and anabolic activity. Anamorelin is currently being investigated in phase III studies for the treatment of anorexia-cachexia in advanced non-small cell lung cancer (also known as the ROMANA program).

\section{Material and Methods}

Eighty-two patients with advanced cancer, ECOG performance score $\leq 2$ and weight loss $5 \%$ within prior 6 months were treated with placebo $(\mathrm{n}=36)$ or $50 \mathrm{mg}$ anamorelin $(n=38)$ once daily for 12 weeks. Body composition by DXA, handgrip strength (HGS) and Quality of Life (QoL; ASAS scale) were assessed at baseline, 4,8 and 12 weeks. Solid tumors represented prevalent malignancy $(>93 \%)$ with no difference in cancer types between treatment groups.

\section{Results}

Treatment with anamorelin for 12 weeks significantly increased total body mass (TBM) and LBM. Increases in

${ }^{3}$ Helsinn Therapeutics, Inc., Bridgewater, New Jersey, USA

Full list of author information is available at the end of the article
LBM and TBM in anamorelin-treated patients were strongly correlated $\left(\mathrm{r}^{2}=0.7249, \mathrm{p}<0.0001\right)$. Anamorelin treatment improvements in HGS and QoL were previously presented [1]. Anamorelin was well tolerated, and types and prevalence of AEs were similar between treatment arms.

\section{Conclusion}

Decreased body weight and LBM are poor prognostic factors in cancer cachexia patients. This study demonstrates that $50 \mathrm{mg}$ anamorelin treatment for 12 weeks significantly increased LBM, which largely contributed to the increases in total body mass. Together with its appetite-enhancing activity, these results support the further development of anamorelin in treating cancer anorexia-cachexia.

\section{Authors' details}

'Department of Medicine, Molecular \& Cellular Biology, and Huffington Center on Aging, Baylor College of Medicine, Houston, Texas, USA. ${ }^{2}$ Center for Translational Research in Inflammatory Diseases, Michael E De Bakey VA Medical Center, Houston, Texas, USA. ${ }^{3}$ Helsinn Therapeutics, Inc., Bridgewater, New Jersey, USA.

Published: 28 May 2014

\section{Reference \\ 1. Garcia JM, Yan Y, Manning Duus E, Friend J: Anamorelin's effects on appendicular lean body mass in cancer patients with cachexia; results from a phase II randomized, double blind, multicenter study. Annals of Oncology 2012, 23(Suppl 9):ix512.}

doi:10.1186/2049-3002-2-S1-P19

Cite this article as: Garcia et al:: Effects of the ghrelin receptor agonist anamorelin on lean body mass in cancer patients with cachexia; results from a Phase II randomized, double blind, multicenter study. Cancer \& Metabolism 2014 2(Suppl 1):P19. 